\title{
Perception of Medical Assistance in Dying Among Asian Buddhists Living in Montreal, Canada
}

OMEGA_Journal of Death and Dying

(C) The Author(s) 2020

Article reuse guidelines: sagepub.com/journals-permissions DOI: $10.1177 / 0030222820948645$ journals.sagepub.com/home/ome @SAGE

\section{Nidup Dorji ${ }^{1,2}$ (D), Sylvie Lapierre ${ }^{1,3}$, and Dolores Angela Castelli Dransart ${ }^{4}$}

\begin{abstract}
In the Western world including Canada, grievous and irredeemable health conditions, which cause unbearable suffering, has given support to the legalization of medical aid in dying (MAiD). It is unknown how Asian Buddhists who are in contact with the Western culture perceive MAiD. In this qualitative study, 16 Asian Buddhists living in Montreal took part in a semi-structured interview. Contrary to general findings in the literature, religious affiliation do not always determine moral stances and practical decisions when it comes to MAiD. Some participants were willing to take some freedom with the doctrine and based their approval of MAiD on the right to self-determination. Those who disapproved the use of MAiD perceived it as causing unnatural death, creating bad karma, and interfering with a conscious death. End-of-life (EoL) care providers have to remain sensitive to each patient's spiritual principles and beliefs to understand their needs and choices for EoL care.
\end{abstract}

\footnotetext{
'Faculty of Human Sciences, Centre for Research and Intervention on Suicide, Ethical Issues and End-ofLife Practices, Université du Québec à Montréal, Canada

${ }^{2}$ Department of Public Health, Faculty of Nursing and Public Health, Khesar Gyalpo University of Medical Sciences of Bhutan

${ }^{3}$ Department of Psychology, Université du Québec à Trois-Rivieres, Canada

${ }^{4}$ School of Social Work Fribourg, HES-SO University of Applied Sciences and Arts Western Switzerland Corresponding Author:

Nidup Dorji, Department of Public Health, Faculty of Nursing and Public Health, Khesar Gyalpo University of Medical Sciences of Bhutan, Thimphu, Bhutan.

Email: nidupdorji.dorji@gmail.com
} 


\section{Keywords}

medical aid in dying, death, dying, Asian, Buddhism, religion, culture, opinion

In the recent decades, a considerable amount of attention was given to end-oflife (EoL) care practices. A variety of options were made available to terminally ill patients with unbearable suffering (Castro et al., 2016). Euthanasia (EU) and physician-assisted suicide (PAS) are two of these options. In both instances, these practices are restricted to competent patients who persistently requested death-hastening options as a solution to end their irredeemable condition. With $\mathrm{EU}$, the physician administers lethal medications to patients in order to relieve their suffering by bringing about their death; while with PAS, the physician usually provides the lethal substances, but the patients have to end their own life (Gerson et al., 2020). EU and PAS are legally practiced in 15 regions of the world, mostly in Western countries like Canada (Castelli Dransart et al., 2019). Issues, such as grievous incurable illnesses, or advanced state of irreversible decline (Beuthin, 2018), which cause unbearable physical and psychological suffering, were common justifications for their legalization in these countries (Castro et al., 2016). However, these are emotionally and ethically challenging subjects (Fontalis et al., 2018) and still remain controversial (Castro et al., 2016).

Euthanasia was legalized in the province of Quebec, Canada, in December 10, 2015 (Bravo et al., 2018), and in the rest of Canada in June 2016 (Pesut et al., 2019). Physician-assisted suicide is not legalized in Quebec, only in the rest of Canada. In Quebec, the term "euthanasia" was deliberately replaced by the expression "medical aid in dying (MAiD)", because of the excessive emotional load associated to the word "euthanasia" (Vachon, 2013). A recent poll, conducted by Ipsos (2020) on behalf of Dying with Dignity Canada with 3,502 Canadians, found that $86 \%$ of the sample supported MAiD, while $13 \%$ opposed it (Ipsos, 2020). Among Non-Christians (Jewish, Hindu, Muslim, or Buddhist), $79 \%$ supported MAiD, while $21 \%$ opposed it. However, no information concerning the perception of MAiD among people from each of these other faiths are available, except for the qualitative study by Larm (2019), which was done with a unique sample of Buddhists living in a monastery. With the recent legalization of MAiD in Canada, it would be necessary to examine the perception of participants from various religious minorities separately, since they are often grouped together under the term "Non-Christians" in the Western world and rarely asked for their opinion. Moreover, limiting data collection to affiliation assumes that there is congruity between beliefs, attendance, 
engagement, and affiliation, which are different concepts (Worthington et al., 2003). This study was undertaken to start to fill up the gap on religious minorities, who are often overlooked by academics, policymakers, and health care professionals. People with Buddhist affiliation were chosen since this religion is steadily growing in Canada while gaining acceptance in the mainstream culture (Harding et al., 2010), and because of the inside knowledge of the main investigator about this religion which could help understand and recruit participants.

Death, dying, and diseases are unavoidable facts of life (Schreiber, 2015) and evoke deep and disturbing emotions (Masel et al., 2012). Although death always existed, its perception, and the associated preferences for EoL care, have changed over time according to cultural and societal values and attitudes (Cottrell \& Duggleby, 2016; Mori et al., 2018). Culture fundamentally shapes how an individual makes meaning out of illness, suffering, death, and dying (Kagawa-Singer \& Blackhall, 2001). Attitude towards death in the Western industrialized countries has been generally characterized by denial and the reluctance to speak about it (Zimmermann, 2012); this could be partly related to high-tech medicalization that gives people excessive hope for recovery, to the segregation of sick and older adults into hospices, and to the bureaucratization and professionalization of death-procedures (Tradii \& Robert, 2019).Dying, once viewed as natural, has become medicalized, unacceptable, and unwelcome today (Waldrop, 2011). Advancement in medical technology has also led to the belief that death can be overcome or can be controlled, fueling society's refusal of the finitude of life (Anderson et al., 2011).

Many factors influence the position towards EU and PAS. Younger age, being divorced or widowed, living alone, and higher education and income, were associated with a favorable attitude towards EU or PAS in older adults (Castelli Dransart et al., 2019). Ageist societal discourse about the increasing burden and cost of the elderly population, as well as the perception to be no longer a useful or valuable member of the society increase the likelihood that older adults would choose a hastened death in a hypothetical terminal illness situation (Castelli Dransart et al., 2019). Furthermore, chronic health conditions (e.g. cancer), excruciating and constant pain, depression, hopelessness, being tired of life, perceived loss of dignity and control, or reduced ability to engage in activities that make their life enjoyable and meaningful, motivate patients' request for EU or PAS (Emanuel, 2017; Onwuteaka-Philipsen et al., 2010; Scherrens et al., 2018). In addition, factors such as poor family cohesion, lack of social support, and the perception of being a burden to others, were associated with a favorable attitude toward EU or PAS or a request for hasten death (Ferrand et al., 2012; Yun et al., 2018). Overall, existential and psychosocial factors which impact the patient's sense of self, dignity, and meaning of life are associated with the wish for hasten death (Rodriguez-Prat et al., 2017). For some authors, hastening death is the solution for relieving suffering when facing death and dying (Castro et al., 2016; Rodriguez-Prat et al., 2017), 
while, according to Sprung et al. (2018), the solutions lie in improving palliative care and social support, and in addressing the physical and psychosocial motives for seeking MAiD.

Official religious doctrinal viewpoints usually consider MAiD morally wrong due to its adherence to the principle of sanctity of life (Cohen et al., 2014), and individuals who give more importance to their religious beliefs in daily life were more likely to disagree with EU or PAS (Castelli Dransart et al., 2019; Lapierre et al., 2018). In Europe, being unaffiliated to a religion or Protestant predicted acceptance of EU or PAS as an EoL care option (Cohen et al., 2014). Suffering is perceived differently in Christianity and Buddhism. For Christians, suffering is perceived as a punishment for sin, and seen as redeemable by a powerful loving god (Hall et al., 2018). In Buddhism, suffering is perceived as an integral part of human life, while ignorance, anger, and attachment are regarded as the root causes of suffering, which can be removed (Lee et al., 2017; Masel et al., 2012). The notion of karma (the principle of cause and effect) is an essential part of Buddhists teachings, and embraces the idea that one's intention and actions influence the present and future life of the individual (Lin \& Yen, 2015). Buddhists use the beliefs in karma and the knowledge of impermanence to cope with adversity (Hall et al., 2018).

Due to the widely accepted principle of non-harm in Buddhism, all forms of assisted death, including MAiD, are usually regarded as killing. Therefore, taking away the life of any beings is highly discouraged even if the act is done with benevolence and compassion (Larm, 2019). In Buddhism, bodily death does not permanently resolve suffering, and taking away one's life creates bad karma not only to self, but also to those assisting the act (Larm, 2019).

With the multiculturalism policy introduced in 1971 in Canada (Berry, 2013), immigrants from various countries brought with them their religion. Immigrants from Asia (e.g. China, Tibet, Thailand, Laos, Cambodia, Vietnam, Sri Lanka, or Korea) often had Buddhism as their religious affiliation (Harding et al., 2010). The effect of acculturation, which refers to the assimilation to another culture (Sam \& Berry, 2010), can affect perceptions of a good death or the attitude toward EoL care. However, little is known in these areas (Mori et al., 2018). Western culture tends to value individualism, independence, self-determination and freedom, whereas Eastern societies place much emphasis on collectivism, interdependency, and familial cohesion. These general difference in values could result in divergence in the perceptions of a good death (Miyashita et al., 2007; Mori et al., 2018). It should be noted that immigrants from Asia tend to remain in the tradition they learned in their country of origin (McCormick, 2013).

The Buddhists of Montreal came from various countries and cultures. To the best of our knowledge, there is no study on how Buddhists, in contact with the Western culture, feel about the recent legalization of MAiD as one of the EoL practices. We believe that the findings from this study could inform EoL care providers about the probable influence of patients' Buddhist beliefs on their perception of death and dying in order to better understand their needs and 
choices for the EoL in the context of MAiD, and provide adequate care especially on a spiritual level.

\section{Method}

\section{Design and Procedures}

This research is an exploratory descriptive study with a qualitative research design, because it can provide more detailed information to explain the perception of MAiD among Asian Buddhists living in a Western culture. Participants had to describe themselves as Buddhist, had to be 18 years of age and over, be resident in the city of Montreal (Canada), and speak English fluently. Participants could be followers of one of the main schools of Buddhism (Theravada, Mahayana, and Vajrayana). There were no specific exclusion criteria in this study.

After receiving the approval of the Research Ethics Committee of the University of Quebec in Trois-Rivières (CER-19-259-07.07), participants were given an information letter that described the goal of the research, their role in the study, the risk and benefits of their involvement, and presented the main topics of the research: death, dying, as well as MAiD, so they could make an informed choice about their participation. No incentives were provided to the participants for their involvement in the study. Potential participants were informed that the interview would be audio-recorded, and that each participant would be assigned a numerical code to ensure anonymity. Those agreeing to take part in the study signed a voluntary informed consent form.

For the recruitment, we first made contacts with various Buddhist centers in Montreal. We provided a short description of the study, requesting them to put it on their website for the potential participants to take part in the study. Unfortunately, that approach did not work. Therefore, the principal investigator contacted two Tibetans who owned a business in Montreal, which in turn helped to contact other potential participants in their catchment area. This kind of sampling strategy is called snowball technique also known as 'chain referral' or 'networking' sampling (Schneider et al., 2016). Owing to the fact that the researcher is a foreigner in Canada, the application of this method for recruitment was found appropriate. The principal investigator also participated in Buddhist activities during weekends, and managed to arrange for interviews with other potential participants, trying to recruit an equal number of men and women, in order to complete this convenient sample. Data were collected between September and November 2019.

A scoping review on MAiD was conducted to assist in the drafting of the 17 open-ended questions used for the semi-structured interview (see Table 1) to comprehensively explore participants' perceptions about death and dying with dignity, as well as their preferences for EoL care options and their opinion about 
Table I. Guidelines for the Semistructured Interview.

I. How do you see aging?

2. How do you see yourself getting old?

3. When you think about death, what comes to your mind?

4. Have you experienced the death of a close family/friend? Can you describe your experience of this situation/event?

5. Have you experienced serious illness or have to ever been close to death at one point of time in your life?

6. What do you know about Karma and reincarnation? Do you believe in it?

7. What do you think will happen after one dies?

8. How would you like to die? What would be a "good death" or "dying well" for you?

9. Do you think that it is possible to prepare for dying? If yes, how? What can someone do to prepare for a good death? What would you do to prepare for your own death? What do you think would help you die peacefully?

10. Do you think your Buddhist beliefs influence the way you see death? How?

II. Do you think your family influence the way you see death? How?

12. What does "dying with dignity" mean to you? How can a person "die with dignity"?

13. Have you heard about medical-aid-in dying? If yes, what did you hear about it?

14. In Canada, medical-aid-in-dying (MAiD) was legalized in 2016 for people who are at the end of their life with no hope for recovery. It means that the doctor can give a lethal substance to the patient to end their life. What is your opinion about it? Would you ask for MAiD for yourself if you were in the same situation (at the end of your life with no hope for recovery)? (suppose you are not able to communicate) Would you be comfortable with a close family member or friend asking MAiD for you?

15. There are various end-of-life care? What would you prefer?

A. Relieve pain (with medications) and discomfort as much as possible, and let the process of death take its natural course (it is called palliative care);

B. Extend life as much as possible even if the process of dying would be longer;

C. Refuse/withdraw treatment;

D. To be put into a state of unconsciousness (deep sedation) to remove intolerable suffering completely.

16. Did you make some specific decisions about your funerals (what would you like?, did you tell your family?) or are you leaving these decisions to your family

17. Do you think your Buddhist beliefs might influence the way you see MAiD. If yes, How? Do you think your family influences the way you see MAiD? How?

MAiD. The latter is the main topic of the present paper. Each participant also completed a short sociodemographic questionnaire. Interviews were conducted face-to-face in a quiet place, and lasted between 45 to 120 minutes.

\section{Participants}

As shown in Table 2, the sample included 8 males and 8 females ranging in age from 26 to 66 years $($ median $=53$ years). The majority $(n=14)$ had at least an 
Table 2. Sociodemographic Characteristics of the Informants.

Sociodemographic characteristics

Number of participants

Gender

Male

8

Female

8

Age (in years)

Median

53.00

Education level

High school

2

Undergraduate

9

Master's degree

1

$\mathrm{PhD}$

Others

Place of education attended

India

Taiwan

Canada

Australia

Employment status

Employed

Unemployed

Retired

Others

Marital status

Married/living de facto

Single

Divorced/separated

Birth place

Canada

Others

India

Nepal

Taiwan

Tibet

Vietnam

What language do you feel more at ease to communicate?

French

English

Tibetan

Mandarin

Vietnamese

Language mostly spoken at home 
Table 2. Continued.

\begin{tabular}{|c|c|}
\hline Sociodemographic characteristics & Number of participants \\
\hline English & 0 \\
\hline Tibetan & 5 \\
\hline Mandarin & 2 \\
\hline Vietnamese & 2 \\
\hline \multicolumn{2}{|l|}{ Language mostly spoken at work? } \\
\hline French & 13 \\
\hline English & I \\
\hline Tibetan & 0 \\
\hline Mandarin & 2 \\
\hline Vietnamese & 0 \\
\hline \multicolumn{2}{|l|}{ Years in Canada } \\
\hline Median (min-max) & $21(1-66)$ \\
\hline \multicolumn{2}{|c|}{ How often do you listen to or read Buddhist teachings? } \\
\hline Everyday & 5 \\
\hline At least once a week & 4 \\
\hline Once in a month & 3 \\
\hline Once in a year or less & 3 \\
\hline Never & I \\
\hline \multicolumn{2}{|c|}{ How often do you conduct Buddhist rituals or practice (e.g. meditation) at home? } \\
\hline Everyday & 10 \\
\hline At least once a week & 0 \\
\hline Once in a month & 3 \\
\hline Once in a year or less & 2 \\
\hline Never & I \\
\hline \multicolumn{2}{|c|}{ How often do you participate in Buddhist rituals (pujas) at a Buddhist centre (temple) } \\
\hline Everyday & 4 \\
\hline At least once a week & I \\
\hline Once in a month & 5 \\
\hline Once in a year or less & 6 \\
\hline Never & 0 \\
\hline \multicolumn{2}{|c|}{ How would you rate your present health condition? } \\
\hline Poor & 0 \\
\hline Fair & 2 \\
\hline Good & 10 \\
\hline Excellent & 4 \\
\hline
\end{tabular}

undergraduate level of education and half $(n=8)$ attended their schooling in Canada. Eleven were married or living with a partner, and currently employed. All, except one, were born outside of Canada. Quebec is a French-speaking region of Canada and 13 of the participants spoke mostly French at work. The median years of stay in Canada was 21 years (range: 1-66 years). Five of 
the participants read Buddhism teaching materials every day, and ten practiced meditation on a daily basis. Nonetheless, participation in Buddhist rituals at a center was less frequent (11 participants mentioned being involved in rituals once a month to once a year). All the participants reported that they did not suffer from any chronic diseases, and 14 self-rated their health condition as good or excellent.

\section{Data Analysis}

There is no specific guideline to determine the sample size necessary for a qualitative research. A common range lies between 8 and 26 (Isaacs, 2014; Schneider et al., 2016). The attainment of saturation is often the guiding principle to assess the adequacy of the size of a purposive sample. It refers to the point in data collection where additional participants don't provide any additional insights (Saunders et al., 2018). At the beginning, we proposed 30 participants to fulfil the objective of this study. However, during the simultaneous collection and transcription of the data, it was apparent around the $11^{\text {th }}$ participant that no more additional insights were emerging. However, within the time and resource available, five more participants were recruited, hoping that new information would be generated on the phenomenon under investigation. Data collection stopped at the $16^{\text {th }}$ participant.

The recorded verbatims were transcribed while repeated and immersive reading led to the identification of recurring themes. The verbatim from each of the 16 participants was considered as the unit of analysis. To organize, elicit meaning, and draw conclusions from the data collected, a content analysis was conducted using NVivo version 12 (Bengtsson, 2016). Analysis first began with the assigning of broad codes to the data, followed by building a second cycle of codes on the initial broad codes, and grouping them into meaningful categories or themes (Kalpokaite \& Radivojevic, 2019). This process is called categorization (Schneider et al., 2016). Data coding was based on the interview guidelines applied for data collection and guided by the research question (Kalpokaite \& Radivojevic, 2019). Throughout the analysis, the research team revisited transcripts until a consensus was drawn about the categorization. Also, throughout this qualitative inquiry, the principal investigator was aware and averted the influence of one's own assumptions and perspective on the interpretation of the research data (Kalpokaite \& Radivojevic, 2019).

\section{Results}

All participants acknowledged having heard about the existence of MAiD at one point of time in their life. However, two of the participants did not know about its legalization in Canada. Half (8/16) of the participants (more among male, 5/ 8) disagreed with MAiD option, especially participants with knowledge about 
Buddhism. While some participants agreed (5/16) with the use of MAiD (more among female, 4/5), three were ambivalent. This section will present the attitudes and standpoints of the interviewees about MAiD, as well as some factors that could have influenced their views, such as their personal experiences with death and dying.

\title{
Disagreement With MAiD
}

Half $(n=8)$ of the participants were not in favor of MAiD, which was perceived as contrary to Buddhist teachings (known as the dharma). A male participant who has been practicing Buddhism for the past 40 years (Pt.16, 54 years), explained: "As Buddhist, MAiD is not a correct option. It is against the precepts of Buddha dharma (teachings). A law is manmade and is liable for change. Buddha clearly mentioned, not to rely on a person, but to rely on the truths the dharma."

The content analysis of the interviews revealed five main themes underlying participants' arguments against the use of MAiD. The reasons included: 1) MAiD is taking the life of a living being $(n=6)$; 2) MAiD creates bad karma for those involved $(\mathrm{n}=8)$; 3) MAiD will relieve suffering only in the present life $(\mathrm{n}=4)$; 4) MAiD disrupts the natural process of dying $(\mathrm{n}=8)$; and 5) MAiD hinders a conscious death $(\mathrm{n}=8)$.

\section{Kind of Killing, No Different From Suicide}

MAiD was perceived as an act of killing a living being, and as such is condemned by Buddhism. Three of the participants considered that MAiD was no different from suicide, since the patients would request death to eliminate their suffering.

\begin{abstract}
"As a Buddhist, I don't agree with it. Whether somebody infuses me the lethal substance or I do it by myself, it is anyway leading a person to die which is not natural. As a Mahayana Buddhist practitioner, killing is condemned and is a heinous crime. That is from a Buddhist view. Even if we look from another view, if you kill yourself, you are not accepting what is happening to you: the death, the suffering." (Male Pt.12, 50 years)

"Coming from Buddhist background I have a strong influence to condemn MAiD. $M A i D$ to me is no different from suicide and taking away life is against the teachings of Buddhism." (Male Pt.15, 59 years)
\end{abstract}

\section{Belief in Karma and Temporary Relief of Suffering}

The belief in karma is common among Buddhists. Any actions that are harmful, either to oneself or to others, such as killing, will create bad karma. Therefore, 
killing will create bad karma for the perpetrator, as well as for the individual requesting MAiD. In addition, suffering in life is perceived as an integral part of human life and as a result of both past and present bad karma. Suffering gives the person the opportunity to practice the removal of desire, anger, attachment and ignorance that lie at the root of suffering. All eight participants opposing MAiD considered that it created bad karma for the patients and those who provide the help to die.

"We believe it takes millions of rebirths to get this fortunate human body. We call it precious human birth and taking away life through MAiD is not different from killing, is against our belief, and prohibited. The person has to face the negative consequences for resorting to such an act." (Female Pt.4, 59 years)

"If sickness that causes pain and suffering is the reason for opting (for) MAiD, I would consider my illness as a result of my bad karma. Sickness is a blessing for me to practice dharma more, and train my mind. If I suffer from a chronic terminal disease like cancer, I would resort to many kinds of alternative therapy such as yoga, Tai Chi, Qi-gong, to come in terms with my illness and improve it." (Female Pt.6, 42 years)

"As a Buddhist, we reap our own karma, and we need to purify our own negative karma, which is not really the case with MAiD. Suffering for us Buddhists is like purifying the negative karma so that in the successive lives, you have exhausted your negative karma and reap better karma." (Female Pt.1, 52 years)

In Buddhism, pain and suffering are considered as perceptions or interpretations of the mind, and MAiD will relieve one's misery only in this present life. It seems more important for Buddhists to pay attention to the cyclical existence of birthdeath-rebirth, exhaustion of bad karma, and attainment of liberation than to relieve current suffering.

"The reality is (that) suffering is a self-constructed phenomenon, which is intangible, has no physical entity, and suffering just exists at the thinking level and is just a belief. It is the mental agitation that makes the person say is suffering [...] MAiD may temporarily end suffering in this life, but your karma will follow you wherever you go and take rebirth." (Male Pt.16, 54 years)

\section{Causing Unnatural Death and Hindering Conscious Death}

All participants who disapproved of MAiD were follower of Tibetan Buddhism and believed that it was an external intervention that induced an unnatural death. MAiD disrupted the natural process of dying. A female participant (Pt.1, 52 years) said: "Death caused by MAiD is not going to be natural. MAiD is 
something that helps end life, but it is against natural death [...] As much as possible, I would opt for natural death to happen in my life." All participants considered that MAiD would interfere with a conscious death, which is important in Tibetan Buddhism, and four of them even emphasized their preference to achieve a mindful death. The latter places a strong emphasis on knowledge of the dying process, that includes eight stages of dissolution. Buddhists are encouraged to contemplate on these stages every day to prepare for the ultimate transition.

"If someone put me off with the help of medication, that would interfere with the importance I give to my conscious death; (it) would be a bad death for me." (Female Pt.6, 42 years)

"Peaceful death is very important. Especially from the Buddhist point of view, it is very important to practice dharma in life, especially towards the end of life and at the time of dying. It is very important to focus on the inner energy. Even if you do good things in whole life and at the time of dying, if something disturbs the departing consciousness, it will lead to have bad things in next life, you know. Dying mindfully is very important. For that, it needs peace and calm, mindful and conscious. No disturbance." (Male Pt.15, 59 years)

\section{Agreement With MAiD}

A third of the sample $(n=5)$ was in favor of MAiD. The content analysis of the interviewees indicated that three themes were mentioned for endorsing MAiD: 1) avoiding unbearable suffering, 2) refusing to be a burden to others, and 3) the right to self-determination, the latter referring to the belief that the person is the only one who can make decisions about one's life and death.

\section{Avoiding Suffering}

Pain and irredeemable chronic health conditions were the main reasons for participants to approve the use of MAiD. Most of those agreeing with this practice were women (4/5). One of them (Pt.5, 38 years) said "I think he or she should be given the chance to go peacefully, rather than to see the person waiting to die going through all kinds of pain and sufferings [...] If I am chronically ill, suffering, and there is no point of revival, why should I not go for it." In the following quotation by the only male participant (Pt.9, 54 years) who supported MAiD, it is clear that religious beliefs did not always determine one's opinion toward MAiD. Even if he generally acknowledges Buddhist's beliefs, his reflections on suffering makes him willing to take some liberties with the religious principles when it comes to EoL care.

"I don't think in Buddhism it is said you should suffer and die without dignity. My understanding of Buddhism is to extend help if you see people suffering. Are you going 
to help or are you going to let them suffer? I don't remember Buddhism telling me if I see another suffering, let them suffer but try to find some ways to help ease suffering. However, if you intend to take away somebody's life, it is against the philosophy of Buddhism [...] But to see people suffer and let them suffer amidst options available make no sense. By resorting (to) MAiD, if I go to hell or take rebirth in the lower realms, so be it. Seeking MAiD will be more practical oriented than philosophical arguments. With the criteria cited for MAiD, and with the choice available, if people are genuinely suffering, I think they should avail the option. This influence is more of my thought process and my lived experience, and being rational in my thinking makes me less Buddhist, then so be it."

\section{Refusing to Be a Burden to Others}

Knowing that one is a burden to others is often painful. Three participants mentioned their refusal of being a problem to others as a reason for their approval of MAiD. They also added that their argument was based more on pragmatism: MAiD was perceived as a mean to solve a problem.

"I don't think my Buddhist philosophy influences this decision. It would rather be the practical aspects of life that influences me to opt for MAiD. Why should I be of burden to anybody including myself? Life has already become too much and too stressful for everybody. I would not prefer to be of burden to anybody. I would rather go peacefully with such (an) intervention. I mean let's be more practical here." (Female Pt.5, 38 years)

In relation to the importance given to practicality, two participants suggested that their perceived lack of solidarity and social support for vulnerable others in the Western world were reasons for favoring MAiD. This opinion relied on the comparison with the Eastern values where social support for the ailing patient is perceived readily available. A female participant (Pt. 13, 30 years), having lived most of her time with Western friends, said:

"I know not every one of us is blessed to have somebody close to us. Otherwise, there is no
reason why we see so many homeless people here. For these people, they lack social
support. If social support can do something more than MAiD, why don't we promote
this? I can understand why MAiD exists. MAiD is certainly a very difficult and a con-
troversial topic [....] At a deeper level, we will have to consider where we as a society are
falling. I don't think anyone in my generation would be able to support a dying person."

"Social support for the dying person is less in this part of the world, where everyone is busy and time is money, MAiD maybe a good option. However, social support is 
readily available in the culture we come from, one of the members of the family are always there to be by the side of the sick person." (Male Pt.10, 26 years)

\section{Self-Determination}

The desire to decide and choose for oneself and the right for self-determination also influenced the opinion about MAiD. Thirteen participants ( 7 males and 6 females) mentioned the topic of individual choice and control over one's life. However, only those who agreed with MAiD considered that they could exercise this right. Those who disagreed with MAiD or who were ambivalent stated that others can use this right but that they would turn down this opportunity for themselves. Four out of five participants (three female and one male) who agreed with MAiD based their approval on the right for self-determination.

"I think he or she should be given the chance to go peacefully, rather than to see the person waiting to die going through all kinds of pain and sufferings ....If I am chronically ill, suffering, and there is no point of revival, why should I not go for it." (Female Pt. 5, 38)

"I cannot have my family's decide MAiD on me. I say I cannot because they won't know what I am going through. Only I would know myself better than anybody would. Better, not involve family here. As far as possible, I will let my decision be mine since my position relates to my case only [...] MAiD is a very complicated topic [...] The best (decision) lies in the hand of the patient. Leave it to them to decide." (Male Pt.10, 26 years)

"If the person is suffering, intensely hard for the family to see, I feel the person has to full right to say if he or she does not want to life anymore." (Female Pt.5, 38 years)

\section{Ambivalence Toward MAiD}

Three of the participants remained ambivalent about the use of MAiD in EoL care. They could understand the sensible and practical aspects of the act, but were confronted by their Buddhist beliefs, and the reaction of their family, as by the uncertainties of the future. Not limiting their answer to "I don't know", their deliberations showed that they had arguments for both approving and rejecting MAiD which reinforced their indecisiveness. MAiD was considered a challenging issue making it difficult to take a stand.

"I don't know what my concrete opinion about MAiD will be. However, I think we will have to put into context, rather than universalize the ideas on moral, ethical, or 
values. If we put into contexts, such as the fact that the dying person is suffering from a terminal illness with no hope of recovery, suffering and the dying person is begging the medical team to cause him or her death, in such case, I think it will be all right to have MAiD. However, it also contradicts the teachings of Buddha. So I don't know really [...] Ultimately it comes down to view (perception) and intention of a person's actions." (Male Pt.10, 26 years)

"I understand MAiD brings peaceful death to a dying person. However, I don't know whether I would prefer it or not. Only time would tell me and, at that moment, I will consider how family members, my relatives, and I feel (about) it. I think I would rather leave it to the future." (Female Pt.4, 59 years)

\section{Factors Influencing Views of MAiD}

In the previous section, we have presented the attitudes and stances of the participants towards MAiD. In the present section, we added some information about the fears and other factors that could have influenced their views of MAiD. Some participants (5/16), were concerned about the possibility that MAiD could be misused. This possibility is often mentioned in Western societies as a reason not to legalize MAiD. A male participant (Pt.10, 26 years) said: "They (people) think life is all about money and power. If we become a victim of power and money, we can hardly think about moral and ethics. In that way, it is sure MAiD can be misused. People will think fellow beings are mere resources instead of considering them as another fellow being. It is very sad."

The culture and family upbringings of the participants is strongly intertwined with the philosophy of Buddhism. Even if many participants $(12 / 16)$ spoked about the influence of their family, culture, and education on MAiD, Buddhists values always mediated their opinion. Ten out of 13 who answered the question on family influence considered that the Buddhist values of their family did guide their perception of MAiD. The question also led participants to talk about the experience they had with the illness and death of a family member, which seemed to have some bearing on their opinion about MAiD. When they were witness to their relatives' suffering, they mostly conceded the entitlement to MAiD.

"I grew up in a family influenced by Buddhist philosophies. However, I will also not forget that I grew up with friends who were mostly Westerner and followers of the Catholic religion, and I can clearly see the difference. Although I don't believe in the doctrine of Buddhism, I think (Western) people tend to see death as a problem which I don't know, and I guess for Catholic followers, death is a moment that decides whether the dead person will ascend to heaven or go to hell depending on what they did. This is something I do not believe in. While death for them is more of a turning point, I see death is more of continuity even though it is terrifying. However, I don't 
have difficulty talking about death, unlike my Western friends." (Female Pt.13, 30 years)

"My late mom suffered from amyotrophic lateral sclerosis (ALS). ALS can let the person maintain mental stability but (is) physically challenging as one function of the body deteriorates over the other until the person cannot breathe any longer and suffocate. Would I dare to see my mom suffer? No! I would rather have the doctor give her something to go peacefully. I would not be able to stand there and watch my mom suffocate and try getting air. To me that is inhuman and in that sense, MAiD is more human." (Male Pt.9, 54years)

\section{Discussion}

This study explored how some Asian Buddhist living in Montreal perceived MAiD, which was legalized recently in Quebec and in Canada. Contrary to results generally found in the literature about the association between religion and the attitude toward MAiD (Castelli Dransart et al., 2019), religious affiliation or beliefs do not always determine moral stances and practical decisions when it comes to MAiD. In our sample, a first group seem to live and want to die according to the Buddhist beliefs and teachings; a second group generally relied on Buddhists philosophy, but was willing to take some freedom with the doctrine when it came to MAiD, and finally, a third group was ambivalent and questioned religious and practical arguments for and against MAiD. These observations confirm results by Larm (2019), who interviewed members of a Canadian Buddhist monastery. She found that, although MAiD contradicts Buddhist teachings, in some circumstances maintaining moral absolutes was less important than respecting the dying person's autonomy and being able to view each case of suffering with wisdom and compassion.

As for sociodemographic characteristics, none seemed to distinguish each group of attitude toward MAiD, except for gender. In contrast to some study findings (Castelli Dransart et al., 2019; Emanuel et al., 2016; Ulas Karaahmetoglu \& Kutahyalioglu, 2019), female participants seem to favor MAiD more in this study. As argued by Mercadillo et al. (2011), compassion, empathetic sensitivity, and manifestation of extra care for others through emotional appraisal, may have influenced female support for MAiD as a way to end suffering. Although higher level of education was consistently associated with higher acceptance of MAiD in previous studies (Castelli Dransart et al., 2019; Smith et al., 2015), our participants disagreed or were ambivalent with this practice, even if the majority had a university degree. However, it is apparent that the attended place of education seemed to influence the approval of MAiD. All five who agreed with MAiD were educated in Western countries (Canada and Australia). Whether immigrants' place of education, especially in the 
countries where MAiD is legalized, influences the endorsement of this practice is worth exploring in future.

Participants who define themselves by their spiritual affiliation ("As a Buddhist, I ...") were less in favor of MAiD. This observation was consistent with most previous studies on the topic, where higher religiosity was a consistent predictor of disapprobation with MAiD (Castelli Dransart et al., 2019; Lapierre et al., 2018). In fact, the deliberate act of hastening death is not supported by any religions, including Buddhism (Larm, 2019). In the teaching of Buddhism, birth as a Human is considered rare and precious, giving the opportunity to practice the dharma which is perceived as the gateway to the liberation from all suffering and to nirvana (Perrett, 1996). Buddhists would lament this missed opportunity and regard it as a great loss (Dhondup, 2007).

As is the Christian religion, where it is forbidden to kill a human life (May, 2003), Buddhist participants who disapproved of MAiD did so because they regard this practice as killing, even if it is done with benevolence and compassion (Larm, 2019). Interestingly, participants had additional reasons to reject MAiD that differed considerably from opponents drawn from various Western sample (Cohen et al., 2014). Among these reasons, they mentioned the creation of bad karma, the importance of achieving a conscious death or dying with awareness, and the perception that suffering is an opportunity form mental training (Lin \& Yen, 2015; Xu, 2019). The information, stressed by the participants, on the importance of achieving a conscious death, or dying with awareness, is unique and not mentioned in the scientific literature.

Buddhists participants also acknowledged ageing, illness, death, as well as suffering as normal aspects of life (Masel et al., 2012). Seven out of eight participants who disagreed with MAiD considered that the solutions for reducing suffering laid more in addressing the reasons for seeking MAiD, dealing with negative emotions, and improving palliative care and social conditions than in hastening death (Sprung et al., 2018). Some participants also clearly regarded MAiD as equivalent to suicide, which is rarely mentioned in Western sample who consider that MAiD is a rational act, unlike suicide, which is related to mental illness (Dembo et al., 2018; Downie \& Dembo, 2016).

Consistent with other studies on the attitude toward MAiD (Beuthin, 2018; Castelli Dransart et al., 2019; Castro et al., 2016; Pestinger et al., 2015), proponents based their claim on self-determination and the relief of suffering, as well as a concern about being a burden to family and friends. When mentioning the latter, Buddhists participants pointed that MAiD endorsement could be culturally contextualized: Asian social obligations for caring for family members are challenged by the Western values of individualism, autonomy, or independence (Mori et al., 2018). It should be mentioned that four of the five participants in favor of MAiD were living in Canada for more than 15 years. This could indicate that exposure to Canadian culture could have shaped how the individuals perceived and made meaning out of illness, suffering, death, and dying 
(Kagawa-Singer \& Blackhall, 2001). However, for our participants, there was no difference between culture and religious beliefs, both are closely connected among Asian Buddhists, which is the opposite for Quebecers who rejected religion to free themselves from the authoritarian influence of the Catholic Church on issues surrounding morality (Lapierre et al., 2018). Furthermore, it was surprising to note that, contrary to Western proponents of MAiD, none of the Buddhist participants in favor of MAiD mentioned loss of dignity or the lack of quality of life (unable to enjoy life's activities), which are frequent reasons for requesting MAiD (Wiebe et al., 2018). It appears that participants coming from a Buddhists background did not associate the loss of quality of life in old age or at the end of life with the request for hastening death probably because in Buddhism the concept of impermanence (nothing lasts) is entrenched as part of life.

\section{Strengths and Limitations of the Study}

This study is the first to investigate the perception of and reaction to the newly legalized law on MAiD in Buddhist individuals' living in Canada. Although, attitude toward euthanasia and dying with dignity has been studied in Asia (for example in Japan in the context of EoL care), surprisingly these studies did not include religious affiliation, such as Buddhism, as an associated variable (Makino et al., 2014; Morita et al., 2004; Okishiro et al., 2009). Moreover, this topic is mostly taboo in Asian societies. Therefore, it is largely overlooked or unaddressed. Another strength of this study is that the participants were provided with a clear definition of MAiD. This is extremely important since there is inaccurate knowledge about these concepts, especially among laypersons, who appear, in other studies, to be confused about the difference between EU, treatment withdrawal and PAS (Marcoux, 2011).

The province of Quebec is mostly a French-speaking region of Canada with $77 \%$ of the population reporting French as its mother tongue (Statistics Canada, 2017). However, in the city of Montreal this proportion drops to $49.6 \%$, since English is the mother tongue of $12 \%$ of the population and other languages are those of $33.3 \%$. Montreal is a multicultural city with many ethnic groups of various religions. It is probably one of the best location in the world to interview people from various Asian Buddhist countries because of this diversity (Arsenault Morin \& Geloso, 2020). Since the principal investigator was fluent only in English, recruitment was conducted only with participants who could communicate in English. However, it was impossible to meet French-speaking Buddhists, and due to this limitation, gaining better insights about MAiD may have been left uncaptured, especially since Francophones in Quebec seem more estranged from religion than the Anglophones (Lapierre et al., 2018). It should also be noted that English was for all of the participants a second or third language, but it did not seem too difficult for them to explain 
their thoughts, since the verbatim quotations are very clear. Recruitment bias is another limitation of the study since this convenient sample was selected through chain referral. Participants might be more similar to each other in their practice of Buddhism, and saturation was probably achieved earlier than with a sample that would include each school of Buddhism. Furthermore, full expression of the participants' opinion may have been suppressed due to the high number of questions in the interview guideline (17 questions). Future study could limit the questions to only one topic to facilitate in-depth discussion.

\section{Future Research and Implications}

At the beginning of this study, we planned to interview 10 participants from each of the three main schools of Buddhism (Theravada, Mahayana, and Vajrayana). However, it was impossible to contact equal numbers of participants from each school of Buddhism, partly because of the lack of contacts within the Thai, Cambodian or Sri Lankan communities (Theravada). Our convenient sample included more Tibetan Buddhists (Vajrayana) and some participants from the Chinese, Taiwanese or Vietnamese communities (Mahayana). Future research should attempt to recruit samples from each school of Buddhism or from various cultural communities where Buddhism is the main religion, in order to capture the possible differences in opinion about MAiD between each of them. Moreover, future studies should look beyond religious affiliation and investigate the views about MAiD according to the participants' perceived level of spiritual commitment, as well as their perception of heavily discussed topics in Buddhism, such as compassion, loving-kindness, or impermanence.

Agreeing with MAiD might have been shaped by personal experiences with illness and death (Okishiro et al., 2009) and this should be further explored in studies with a Buddhist population. While the acceptance of suffering may be prevalent in traditional Buddhist societies, the outlook on adversity and distress may have been changed due to acculturation. New research on the influence of acculturation (Mori et al., 2018) seem to be justified since some of the participants witnessed marked difference in perception towards death and EoL care between their friends from the Western and Eastern culture. Furthermore, since some Asian tend to remain faithful to their cultural traditions (McCormick, 2013), the influence of enculturation on the perception of MAiD would also be interesting.

These results have important implications for health care professionals, especially EoL care providers. Religious affiliation provides general information about the needs of patients and family when a loved one is facing the EoL. However, exposure to Canadian culture, moral principles, and personal and family values, can shape individuals' perception of illness, suffering, death, and dying. Each person is unique and professionals should take the time to 
explore and investigate patients' beliefs and preferences for available EoL practices in order to support them in this last part of life.

\section{Conclusion}

MAiD is an emotionally and ethically challenging issue (Fontalis et al., 2018). Interviews with Asian Buddhists living in Montreal showed that their spiritual beliefs were significant guiding features of their opinion about MAiD, but did not determine if people were in favor or not with MAiD, as observed by Larm (2019). Other factors than religious affiliation, such as personal experiences with the illness and death of a close family members, acculturation, or religious commitment could be relevant. However, since beliefs have a profound impact on how an individual sees life, suffering, and death, it is important that EoL care providers remain sensitive to each patient's spiritual principles and their influence on their preferences for care at the end of their life.

\section{Acknowledgments}

We would like to thank all the participants of this study for their availability and openness, Professor Brian Mishara, and the Centre for Research and Intervention on Suicide, Ethical Issues and End-of-Life Practices (CRISE) for the financial support.

\section{Declaration of Conflicting Interests}

The author(s) declared no potential conflicts of interest with respect to the research, authorship, and/or publication of this article.

\section{Funding}

The author(s) received no financial support for the research, authorship, and/or publication of this article.

\section{ORCID iD}

Nidup Dorji (D) https://orcid.org/0000-0001-6243-0020

\section{References}

Anderson, K. A., Sielski, C. L., Miles, E. A., \& Dunfee, A. V. (2011). Gardens of stone: Searching for evidence of secularization and acceptance of death in grave inscriptions from 1900-2009. OMEGA—Journal of Death and Dying, 63(4), 359-371.

Arsenault Morin, A., \& Geloso, V. (2020). Multilingualism and the decline of French in Quebec. Journal of Multilingual and Multicultural Development, 41(5), 420-412. https://doi.org/10.1080/01434632.2019.1676250

Bengtsson, M. (2016). How to plan and perform a qualitative study using content analysis. NursingPlus Open, 2, 8-14. https://doi.org/10.1016/j.npls.2016.01.001 
Berry, J. W. (2013). Research on multiculturalism in Canada. International Journal of Intercultural Relations, 37(6), 663-675. https://doi.org/10.1016/j.ijintrel.2013.09.005

Beuthin, R. (2018). Cultivating compassion: The practice experience of a medical assistance in dying coordinator in Canada. Qualitative Health Research, 28(11), 1679-1691. https://doi.org/10.1177/1049732318788850

Bravo, G., Rodrigue, C., Arcand, M., Downie, J., Dubois, M.-F., Kaasalainen, S., Hertogh, C. M., Pautex, S., \& Van den Block, L. (2018). Are informal caregivers of persons with dementia open to extending medical aid in dying to incompetent patients? Findings from a survey conducted in Quebec, Canada. Alzheimer Disease and Associated Disorders, 32(3), 247-254. https://doi.org/10.1097/WAD.0000000000000238

Castelli Dransart, D. A., Lapierre, S., Erlangsen, A., Canetto, S. S., Heisel, M., Draper, B., . . Wyart, M. (2019). A systematic review of older adults' request for or attitude toward euthanasia or assisted-suicide. Aging \& Mental Health, 1-11. https://doi.org/ 10.1080/13607863.2019.1697201

Castro, M. P. R. d., Antunes, G. C., Marcon, L. M. P., Andrade, L. S., Rückl, S., \& Andrade, V. L. Â. (2016). Euthanasia and assisted suicide in Western countries: A systematic review. Revista Bioética, 24(2), 355-367.

Cohen, J., Van Landeghem, P., Carpentier, N., \& Deliens, L. (2014). Public acceptance of euthanasia in Europe: A survey study in 47 countries. International Journal of Public Health, 59(1), 143-156. https://doi.org/10.1007/s00038-013-0461-6

Cottrell, L., \& Duggleby, W. (2016). The good death: An integrative literature review. Palliative \& Supportive Care, 14(6), 686-712. https://doi.org/10.1017/ S1478951515001285

Dembo, J., Schuklenk, U., \& Reggler, J. (2018). "For their own good": A response to popular arguments against permitting medical assistance in dying (MAID) where mental illness is the sole underlying condition. Canadian Journal of Psychiatry, 63(7), 451-456. https://doi.org/10.1177/0706743718766055

Dhondup, Y. (2007). This precious life. Tibet Journal, 32(4), 75-76.

Downie, J., \& Dembo, J. (2016). Medical assistance in dying and mental illness under the new Canadian law. Journal of Ethics in Mental Health, 1, 1-9.

Emanuel, E. J. (2017). Euthanasia and physician-assisted suicide: Focus on the data. The Medical Journal of Australia, 206(8), 339-340.

Emanuel, E. J., Onwuteaka-Philipsen, B. D., Urwin, J. W., \& Cohen, J. (2016). Attitudes and practices of euthanasia and physician-assisted suicide in the United States, Canada, and Europe. Journal of American Medical Association, 316(1), 79-90. https://doi.org/10.1001/jama.2016.8499

Ferrand, E., Dreyfus, J.-F., Chastrusse, M., Ellien, F., Lemaire, F., \& Fischler, M. (2012). Evolution of requests to hasten death among patients managed by palliative care teams in France: A multicentre cross-sectional survey. European Journal of Cancer (Oxford, England: 1990), 48(3), 368-376. https://doi.org/https://doi.org/10. 1016/j.ejca.2011.09.020

Fontalis, A., Prousali, E., \& Kulkarni, K. (2018). Euthanasia and assisted dying: What is the current position and what are the key arguments informing the debate? Journal of the Royal Society of Medicine, 111(11), 407-413.https://doi.org/10.1177/0141076818803452

Gerson, S. M., Preston, N. J., \& Bingley, A. F. (2020). Medical maid in dying, hastened death, and suicide: A qualitative study of hospice professionals' experiences from 
Washington state. Journal of Pain and Symptom Management, 59(3), 679-686.e671. https://doi.org/10.1016/j.jpainsymman.2019.10.021

Hall, M. E. L., Shannonhouse, L., Aten, J., McMartin, J., \& Silverman, E. J. (2018). Religion-specific resources for meaning-making from suffering: Defining the territory. Mental Health, Religion \& Culture, 21(1), 77-92. https://doi.org/10.1080/13674676. 2018.1448770

Harding, J. S., Hori, V. S., \& Soucy, A. (2010). Wild geese: Buddhism in Canada. McGillQueen's Press-MQUP.

Ipsos. (2020). Support for medically-assisted dying in Canada. https://d3n8a8pro7vhmx. cloudfront.net/dwdcanada/pages/4493/attachments/original/1582044484/Ipsos_Reid_ DWDC_findings_with_data_charts_Feb_4_2020.pdf?1582044484

Isaacs, A. $\overline{\mathbf{N}}$. (2014). An overview of qualitative research methodology for public health researchers. International Journal of Medicine and Public Health, 4(4), 318.

Kagawa-Singer, M., \& Blackhall, L. J. (2001). Negotiating cross-cultural issues at the end of life: You got to go where he lives. Journal of American Medical Association, 286(23), 2993-3001.

Kalpokaite, N., \& Radivojevic, I. (2019). Demystifying qualitative data analysis for novice qualitative researchers. The Qualitative Report, 24(13), 44-57.

Lapierre, S., Castelli Dransart, D. A., St-Amant, K., Dubuc, G., Houle, M., Lacerte, M.M., \& Maggiori, C. (2018). Religiosity and the wish of older adults for physicianassisted suicide. Religions, 9(3), 66.

Larm, J. (2019). Good deaths: Perspectives on dying well and on medical assistance in dying at Thrangu Monastery Canada. Religions, 10(2), 70.

Lee, K. C., Oh, A., Zhao, Q., Wu, F.-Y., Chen, S., Diaz, T., \& Ong, C. K. (2017). Buddhist counseling: Implications for mental health professionals. Spirituality in Clinical Practice, 4(2), 113-128. https://doi.org/10.1037/scp0000124

Lin, C. -T., \& Yen, W. -H. (2015). On the naturalization of karma and rebirth. International Journal of Dharma Studies, 3(1), 6. https://doi.org/10.1186/s40613-0150016-2

Makino, J., Fujitani, S., Twohig, B., Krasnica, S., \& Oropello, J. (2014). End-of-life considerations in the ICU in Japan: Ethical and legal perspectives. Journal of Intensive Care, 2(1), 9. https://doi.org/10.1186/2052-0492-2-9

Marcoux, I. (2011). Euthanasia: A confounding and intricate issue. In J. Kuře (Ed.), The good death: Controversy in humans and animals (pp. 45-62). https://jemh.ca/issues/v9/ documents/JEMH_Open-Volume_Benchmark_Medical_Assistance_in_Dying_ and_Mental_Illness_Under_the_New_Canadian_Law-Nov2016.pdf

Masel, E. K., Schur, S., \& Watzke, H. H. (2012). Life is uncertain death is certain Buddhism and palliative care. Journal of Pain and Symptom Management, 44(2), 307-312. https://doi.org/10.1016/j.jpainsymman.2012.02.018

May, A. T. (2003). Physician-assisted suicide, euthanasia, and Christian bioethics: Moral controversy in Germany. Christian Bioethics, 9(2-3), 273-283. https://doi.org/10.1076/ chbi.9.2.273.30279

McCormick, A. J. (2013). Buddhist ethics and end-of-life care decisions. Journal of Social Work in End-of-Life \& Palliative Care, 9(2-3), 209-225. https://doi.org/10.1080/ 15524256.2013.794060 
Mercadillo, R. E., Diaz, J. L., Pasaye, E. H., \& Barrios, F. A. (2011). Perception of suffering and compassion experience: Brain gender disparities. Brain and Cognition, 76(1), 5-14. https://doi.org/10.1016/j.bandc.2011.03.019

Miyashita, M., Sanjo, M., Morita, T., Hirai, K., \& Uchitomi, Y. (2007). Good death in cancer care: A nationwide quantitative study. Annals of Oncology: Official Journal of the European Society for Medical Oncology, 18(6), 1090-1097. https://doi.org/10.1093/ annonc/mdm068

Mori, M., Kuwama, Y., Ashikaga, T., Parsons, H. A., \& Miyashita, M. (2018). Acculturation and perceptions of a good death among Japanese Americans and Japanese living in the US. Journal of Pain and Symptom Management, 55(1), 31-38. https://doi.org/10.1016/j.jpainsymman.2017.08.010

Morita, T., Sakaguchi, Y., Hirai, K., Tsuneto, S., \& Shima, Y. (2004). Desire for death and requests to hasten death of Japanese terminally ill cancer patients receiving specialized inpatient palliative care. Journal of Pain and Symptom Management, 27(1), 44-52. https://doi.org/10.1016/j.jpainsymman.2003.05.001

Okishiro, N., Miyashita, M., Tsuneto, S., Sato, K., \& Shima, Y. (2009). The Japan hospice and palliative care evaluation study (J-HOPE study): Views about legalization of death with dignity and euthanasia among the bereaved whose family member died at palliative care units. The American Journal of Hospice \& Palliative Care, 26(2), 98-104. https://doi.org/10.1177/1049909108327027

Onwuteaka-Philipsen, B. D., Rurup, M. L., Pasman, H. R. W., \& van der Heide, A. (2010). The last phase of life: Who requests and who receives euthanasia or physicianassisted suicide? Medical Care, 48(7), 596-603. https://doi.org/10.1097/MLR. 0b013e3181dbea75

Perrett, R. W. (1996). Buddhism, euthanasia and the sanctity of life. Journal of Medical Ethics, 22(5), 309-313.

Pestinger, M., Stiel, S., Elsner, F., Widdershoven, G., Voltz, R., Nauck, F., \& Radbruch, L. (2015). The desire to hasten death: Using grounded theory for a better understanding "when perception of time tends to be a slippery slope." Palliative Medicine, 29(8), 711-719.

Pesut, B., Thorne, S., Stager, M. L., Schiller, C. J., Penney, C., Hoffman, C., Greig, M., \& Roussel, J. (2019). Medical assistance in dying: A review of Canadian nursing regulatory documents. Policy, Politics \& Nursing Practice, 20(3), 113-130. https:// doi.org/10.1177/1527154419845407

Rodriguez-Prat, A., Balaguer, A., Booth, A., \& Monforte-Royo, C. (2017). Understanding patients' experiences of the wish to hasten death: An updated and expanded systematic review and Meta-ethnography. BMJ Open, 7(9), e016659. https://doi.org/10.1136/bmjopen-2017-016659

Sam, D. L., \& Berry, J. W. (2010). Acculturation: When individuals and groups of different cultural backgrounds meet. Perspectives on Psychological Science: A Journal of the Association for Psychological Science, 5(4), 472-481.

Saunders, B., Sim, J., Kingstone, T., Baker, S., Waterfield, J., Bartlam, B., Burroughs, H., \& Jinks, C. (2018). Saturation in qualitative research: Exploring its conceptualization and operationalization. Quality \& Quantity, 52(4), 1893-1907. https://doi.org/ 10.1007/s11135-017-0574-8

Scherrens, A.-L., Roelands, M., Van den Block, L., Deforche, B., Deliens, L., \& Cohen, J. (2018). What influences intentions to request physician-assisted euthanasia or 
continuous deep sedation? Death Studies, 42(8), 491-497. https://doi.org/10.1080/ 07481187.2017.1386734

Schneider, Z., Whitehead, D., LoBiondo-Wood, G., \& Haber, J. (2016). Nursing and midwifery research: Methods and appraisal for evidence-based practice (5th ed.). Chatswood, NSW, Australia : Elsevier Australia.

Schreiber, J. (2015). What makes for a good or bad death? ONS Connect, 30(2), 10.

Smith, K. A., Harvath, T. A., Goy, E. R., \& Ganzini, L. (2015). Predictors of pursuit of physician-assisted death. Journal of Pain and Symptom Management, 49(3), 555-561. https://doi.org/10.1016/j.jpainsymman.2014.06.010

Sprung, C. L., Somerville, M. A., Radbruch, L., Collet, N. S., Duttge, G., Piva, J. P., Antonelli, M., Sulmasy, D. P., Lemmens, W., \& Ely, E. W. (2018). Physician-assisted suicide and euthanasia: Emerging issues from a global perspective. Journal of Palliative Care, 33(4), 197-203. https://doi.org/10.1177/0825859718777325

Statistics Canada. (2017). Montréal [census metropolitan area], Quebec and Quebec [province] (table). Census profile. 2016 census (Statistics Canada catalogue no. 98316-X2016001). https://www12.statcan.gc.ca/census-recensement/2016/dp-pd/prof/ index.cfm?

Tradii, L., \& Robert, M. (2019). Do we deny death? II. Critiques of the death-denial thesis. Mortality, 24(4), 377-312. https://doi.org/10.1080/13576275.2017.1415319

Ulas Karaahmetoglu, G., \& Kutahyalioglu, N. S. (2019). Attitudes toward euthanasia among Turkish university students. OMEGA-Journal of Death and Dying, 79(2), 174-190. https://doi.org/10.1177/0030222817729616

Vachon, M. (2013). Quebec proposition of medical aid in dying: A palliative care perspective. International Journal of Law and Psychiatry, 36(5-6), 532-539. https://doi. org/10.1016/j.ijlp.2013.09.001

Waldrop, D. P. (2011). Denying and defying death: The culture of dying in 21st century America. The Gerontologist, 51(4), 571-576. https://doi.org/10.1093/geront/gnr076

Wiebe, E., Shaw, J., Green, S., Trouton, K., \& Kelly, M. (2018). Reasons for requesting medical assistance in dying. Canadian Family Physician, 64(9), 679.

Worthington, E. L., Wade, N. G., Hight, T. L., Ripley, J. S., McCullough, M. E., Berry, J. W., Schmitt, M. M., Berry, J. T., Bursley, K. H., \& O'Connor, L. (2003). The religious commitment inventory-10: Development, refinement, and validation of a brief scale for research and counseling. Journal of Counseling Psychology, 50(1), 84-96.

$\mathrm{Xu}$, J. (2019). The lived experience of Buddhist-oriented religious coping in late life: Buddhism as a cognitive schema. Journal of Health Psychology, 135910531988274. https://doi.org/10.1177/1359105319882741

Yun, Y. H., Kim, K.-N., Sim, J.-A., Yoo, S. H., Kim, M., Kim, Y. A., Kang, B. D., Shim, H.-J., Song, E.-K., Kang, J. H., Kwon, J. H., Lee, J. L., Nam, E. M., Maeng, C. H., Kang, E. J., Do, Y. R., Choi, Y. S., \& Jung, K. H. (2018). Comparison of attitudes towards five end-of-life care interventions (active pain control, withdrawal of futile life-sustaining treatment, passive euthanasia, active euthanasia and physicianassisted suicide): A multicentred cross-sectional survey of Korean patients with cancer, their family caregivers, physicians and the general Korean population. BMJ Open, 8, e020519. https://doi.org/10.1136/bmjopen-2017-020519 
Zimmermann, C. (2012). Acceptance of dying: A discourse analysis of palliative care literature. Social Science \& Medicine (1982), 75(1), 217-224. https://doi.org/10. 1016/j.socscimed.2012.02.047

\section{Author Biographies}

Nidup Dorji, PhD, is a senior lecturer at the Department of Public Health and Allied Sciences, Faculty of Nursing and Public Health, Khesar Gyalpo University of Medical Sciences of Bhutan. He has been teaching public health to both pre and in-service healthcare professionals in Bhutan for the past 14 years. Besides, he also conducts research, seminars and workshops in the area of adverse life events, quality of life, wellbeing and happiness.

Sylvie Lapierre, $\mathrm{PhD}$, is a full professor in the Department of psychology at the University of Quebec in Trois-Rivieres, Canada. She currently is the co-chair of the Special Interest Group on Suicide Among Older Adults at the International Association for Suicide Prevention. She is also a researcher at the Centre for Research and Intervention on Suicide, Ethical Issues and End-of-life Practices (CRISE) in Montreal, Canada.

Dolores Angela Castelli Dransart, $\mathrm{PhD}$, is a professor at the HES-SO/University of Applied Sciences and Art Western Switzerland, at the School of Social Work in Fribourg. She has an extensive experience in investigating sensitive topics such as suicidality, suicide, grief, bereavement, life events and end-of-life decisions. 